\title{
Mathematical modelling of nano-scaled structures, devices and materials
}

\author{
BARRY JAMES COX
}

(Received 9 October 2007)

Problems from three specific areas of nanotechnology are examined in this thesis. Firstly, new models for nanostructures of carbon are presented. The structures of primary interest are carbon nanotubes and related nanoscale toroidal molecules of pure carbon. Following this, the mechanics of carbon nanotubes interacting with atoms and fullerenes is examined. Acted on by van der Waals forces alone, a fullerene can oscillate at high frequencies inside an open-ended carbon nanotube. The frequencies obtained are predicted to be in the gigahertz range and these so-called 'gigahertz oscillators' provide the primary motivation for this part of the thesis. Lastly, the electrostatic problem of uncharged particles at close interstice subject to an external applied electric field is explored. This is a problem of interest for a class of materials called 'electrorheological fluids' and various aspects of particle geometry are examined.

In this thesis the conventional theory which prescribes the geometric parameters of carbon nanotubes is examined and it is found that it does not adequately account for the curvature inherent in the structure of nanotubes. By considering three postulates and then applying geometry and trigonometry, a polyhedral model is proposed and new formulae are derived which provide more precise expressions for geometric parameters such as the chiral angle, radius and unit length. Asymptotic expansions of these expressions are also shown to produce the conventional formulae as the leading order terms. Following this, approximately toroidal molecules are described which are formed from carbon nanotubes which have defects that cause a bend in an otherwise straight nanotube. The angle of the bend is determined and then expressions are derived giving the dimensions for tori formed from a number of these bend defects. Representative formulae for the ideal tori parameters, of generating radius and tube radius, are determined from integral expressions for the circumference and surface area of the ideal surface.

Thesis submitted to The University of Wollongong, June 2007. Degree approved, September 2007. Supervisors: Professor J. M. Hill and Dr N. Thamwattana.

(C) 2008 Australian Mathematical Society 0004-9727/08 \$A2.00+0.00 
The part of this thesis concerned with the mechanics of carbon nanotubes and fullerenes is primarily focussed on the van der Waals interactions between these types of molecules. The particular application of fullerenes oscillating inside carbon nanotubes is examined in detail and the terms 'suction energy' and 'acceptance energy' are introduced here for the first time to describe the general behaviour of such systems. Following this, the equilibrium offset position for a fullerene inside a carbon nanotube is determined as a function of the radius and the oscillatory motion is modelled using Dirac delta functions, which enables an expression for the frequency of oscillation to be given. This method is extended from spherical fullerenes to those of a spheroidal shape and then extended further to a semi-analytical method which gives the interaction between a carbon nanotube and any molecule which can be modelled as an arbitrary surface of revolution.

Electrorheological (ER) fluids are a class of material which undergo a dramatic change in viscosity when an external electric field is applied. In this thesis an overview of the various theories used to model this induced force of attraction is given, including a brief outline of the major mathematical results and a discussion of the strengths and weaknesses of the various methods. In particular, a detailed description of the re-expansion method is given which is then used to produce solutions to three specific problems. The first problem is that of particles of the same size (i.e. a monodisperse ER fluid) at close interstice and it is demonstrated that the re-expansion method provides a solution that is more congruent with experimental results than a number of other methods. Following this, the method is expanded to the problem of a bidisperse ER fluid comprising non-identical particles. In the analysis of this solution new genuine maxima are found for various ratios of particle size, dielectric constants and particle spacing. Lastly, the method is applied to non-identical conducting particles with dielectric coatings and a solution for this geometry is derived. Analysing this final solution in various limits also derives a useful approximation for thinly coated conducting particles.

In summary, the major original contributions contained in this thesis are: a new polyhedral model for the structure of carbon nanotubes; a novel method for determining the bend angle in polyhedral nanotori and the prediction of new toroidal forms of carbon; the concepts of suction and acceptance energy and a new simple description of the mechanics of gigahertz oscillators; an innovative method to calculate the interaction energy for surfaces of revolution; the discovery of new genuine maxima in the force of attraction for bidisperse ER fluids; and a new solution for conducting particles with dielectric coatings, which leads to an innovative approximation for thin coatings.

\section{BARRY JAMES COX, School of Mathematics and Applied Statistics, University of Wollongong, New South Wales 2522, Australia e-mail: barryc@uow.edu.au}

